\title{
INFLUENCE OF PRECIPITATE MICROSTRUCTURE ON FLOW AND FORMING PROPERTIES OF A 7075 ALUMINUM ALLOY SHEET
}

\author{
F. BARLAT and A. K. VASUDEVAN* \\ Alcoa Laboratories, Alcoa Center, PA 15069, USA \\ *Office of Naval Research, Code-1216, Arlington, VA 22217-5000, USA
}

\section{INTRODUCTION}

It has been shown that precipitates can, depending on their morphology, significantly modify the plastic anisotropy of single crystals ${ }^{1}$ and polycrystals ${ }^{2,3}$. In the present work, the effect of precipitate structure on flow and forming properties of alloy 7075 sheet is investigated. The experiment was designed to evaluate two microstructures having different precipitate features (underaged and overaged conditions) and yet having the same crystallographic texture and yield stress. Several mechanical tests were performed for both material conditions to compare the macroscopic and microscopic responses. In addition, the plastic flow of the 7075 sheets was modeled using the Taylor $4 /$ Bishop and Hill ${ }^{5}$ theory for polycrystalline aggregates. Since this theory does not account for precipitates, the predicted deformation behavior was the same for both underaged and overaged conditions. The difference between the theoretical and experimental results were discussed in terms of how the microstructural features affect plastic deformation in this alloys.

\section{MICROSTRUCTURE}

Alloy 7075 sheet $(\sim 1.6 \mathrm{~mm}$ thick) was cold rolled up to a thickness of $0.76 \mathrm{~mm}$ and fully annealed 2 hours at $413^{\circ} \mathrm{C}$. Then, this sheet was solution heat treated (SHT) for 30 minutes at $466^{\circ} \mathrm{C}$ and quenched in cold water. Standard uniaxial tension specimens as well as plane strain tension specimens taken at $0^{\circ}$ and $90^{\circ}$ to the rolling direction were machined prior to the heat treatments. Specimens were machined and gridded with $\mathbf{2 . 5} \mathbf{m m}$ circles in order to experimentally determine the forming limit curve. Grids used for this work were able to resist a moderate increase of temperature needed for aging the specimens. At this point, half of the material was aged for 25 minutes at $121^{\circ} \mathrm{C}$ leading to an underaged (UA) condition, while the other half was aged for 48 hours at $177^{\circ} \mathrm{C}$ leading to an overaged (OA) condition such that the yield stresses $(0.2 \%$ offset) in the rolling direction were nearly constant ( $300 \mathrm{MPa})$. All of the specimens were kept in a freezer until experiments were performed, in order to avoid any room temperature aging. Thin foils of these two microstructures, prepared by using a double-jet electropolishing technique, were examined in a Phillips 420 electron microscope. The bright field transmission electron micrographs of the UA and OA microstructures are shown in Fig. 1. The UA material exhibits extremely fine GP-zones and some $\eta$ ' precipitates which are not apparent on Fig. 1 because of their small size $(-20 \AA)$. In addition, since the aging time of the UA sample was only $25 \mathrm{~min} / 121^{\circ} \mathrm{C}$, it is quite likely that there is 
some $\mathrm{Zn}, \mathrm{Mg}$ and $\mathrm{Cu}$ in solid solution. On the other hand, the $\mathrm{OA}$ microstructure contains predominantly coarse $\eta^{\prime}$ and $\eta$ precipitates. The $\eta$ precipitates can also be seen at the grain boundaries. While an extremely small precipitate free zone (PFZ) was observed in the UA condition (difficult to identify in Fig. 1), the OA material showed a wide PFZ having a total width of $\sim 0.19 \mu \mathrm{m}$. The orientation relationships of $\eta^{\prime}$ are given by $(0001) \|(111)$ [plane], $(10 \overline{1} 0) \|<110>$ (direction) (see Ref. 6, Table II). X-ray Guinier-de-Wolfe analysis coupled with optical metallography, revealed uniformly distributed intermetallic particles of $\mathrm{Al}_{7} \mathrm{Cu}_{2} \mathrm{Fe}$ and $\mathrm{Mg}_{2} \mathrm{Si}$ constituent phases, a small amount of E-phase $\mathrm{Al}_{12} \mathrm{Mg} 2 \mathrm{Cr}$ dispersoid, and a trace of $\mathrm{S}$-Phase $\mathrm{Al}_{2} \mathrm{CuMg}$.
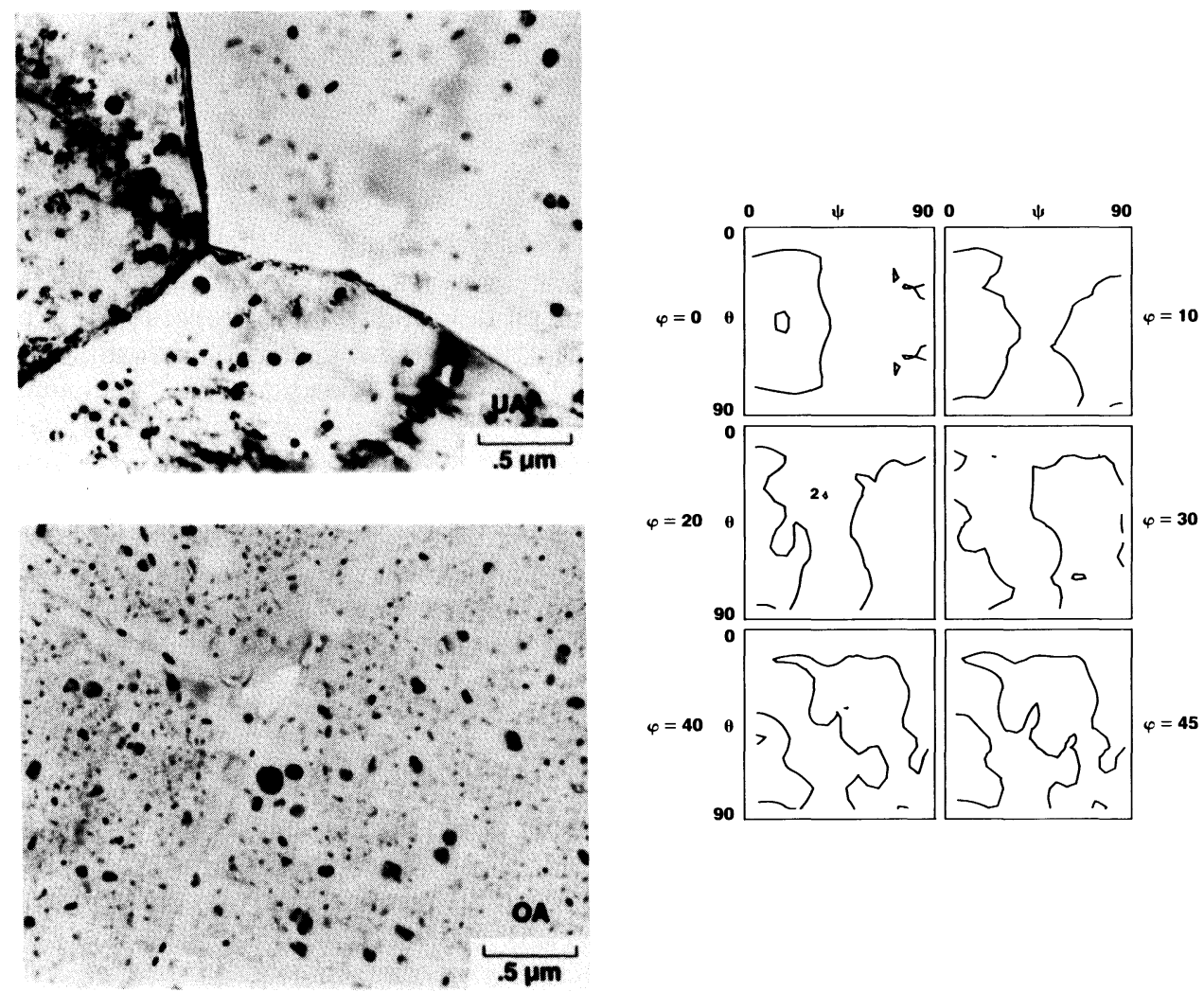

Figure 1. Precipitate microstructure of 7075 alloy underaged and overaged.

Figure 2. Crystallite orientation distribution function (CODF) for 7075 alloy.

The (111) and (200) pole figures determined by X-ray diffraction techniques were used to calculate the crystallite orientation distribution function (CODF) of the 7075 sheet. Fig. 2 represents the CODF intensity of the 7075 sheet for different constant $\phi$ sections in the Euler space defined by $\psi, \theta, \phi$. This plot shows that there is no preferred orientation, since all of the intensity contours are equal to 1 except where indicated in the section corresponding to $\phi=20^{\circ}$, for which a very limited range of orientations exhibit a CODF intensity larger than 2 . When a material exhibits such a random texture, the plastic behavior is expected to be nearly isotropic, at least for moderate plastic strain where grain rotations are small. 


\section{YIELD STRESS AND WORK-HARDENING}

Standard uniaxial tension tests were conducted to determine $0.2 \%$ offset yield stress. Extensometers were used to measure strains in both the loading and width directions of the specimens at all strain levels. The R-value, defined experimentally as the width to thickness strain rate ratio,

$$
R=\varepsilon_{22} / \varepsilon_{33}
$$

was derived from the slope of the true width strain - true length strain plot assuming no volume change occurred in the tensile specimen. Hydraulic bulge tests were conducted for the 7075 sheet in both material conditions. The $0.2 \%$ offset yield stress was obtained by back extrapolation of the stress-strain curve. All uniaxial and biaxial stress-strain curves were approximated by:

$$
\bar{\sigma}=A-B \exp (-C \bar{\varepsilon})
$$

where $\bar{\sigma}$ and $\bar{\varepsilon}$ are effective stress and strain, respectively and A, B and C are material constants. Duplicate $0^{\circ}$ and $90^{\circ}$ plane strain tension tests were performed using the specimen geometry described by Wagoner ${ }^{7}$ for which the width of the specimen gage section is larger than its length (tension direction).
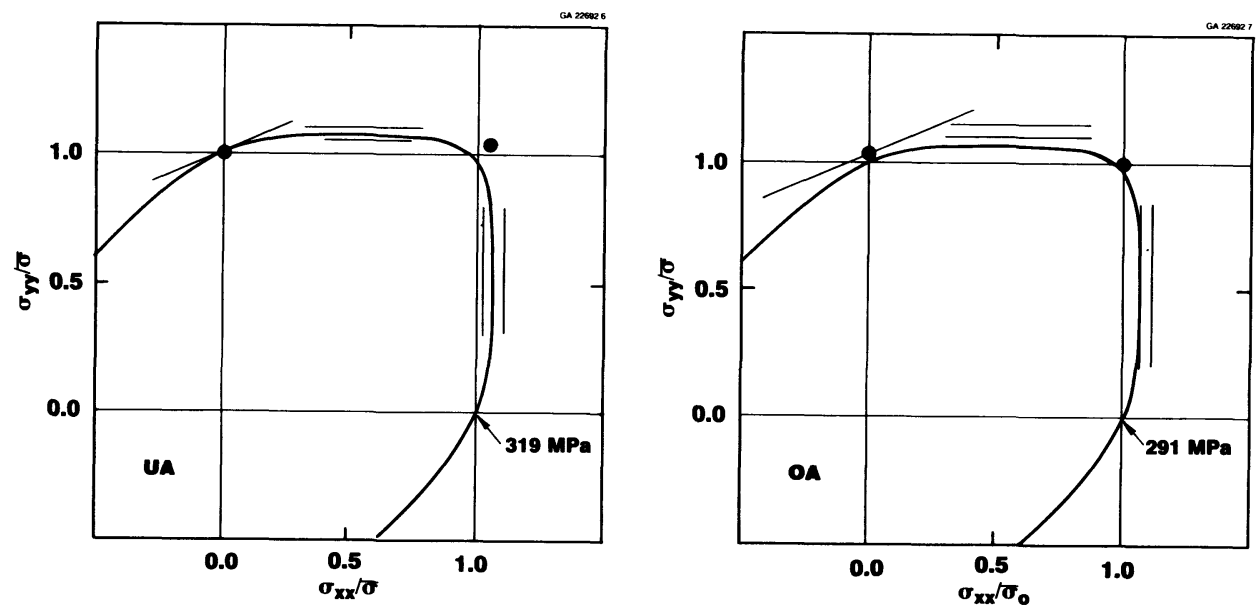

Figure 3. Predicted Taylor/Bishop and Hill (TBH) yield surface for 7075 with experimental points.

Fig. 3 shows the yield surface of 7075 calculated with the Taylor / Bishop and Hill model (TBH) for polycrystalline plasticity. On these plots, $\sigma_{\mathrm{xx}}$ and $\sigma_{\mathrm{yy}}$ represent the stresses in the rolling and the transverse directions, respectively. This model, which is based only on crystallographic texture, predicts the same yield surface for both alloy conditions. However, experimental data points shown on Fig. 3 for the UA and OA alloys, respectively, are in partial agreement with the TBH model. The experimental yield stresses for uniaxial tension along the transverse (TD) direction and for balanced biaxial tension are represented by a filled circle. For uniaxial tension in the rolling 
direction, the tangent to the yield surface can be related to the $R$ value. These tangents have also been plotted in Fig. 3. The duplicate yield stresses in plane strain tension, either in the RD or in the TD, are represented by two lines that are parallel to the $\sigma_{y y}$ or $\sigma_{\mathrm{xx}}$ axes and are, in theory, tangent to the yield surfaces. Actually, these plane strain results show that there is some variability in the yield stress determination. This is not surprising, because experimental determination of the yield surfaces is quite difficult. Perhaps, the salient trend that emerges from these two yield surfaces is the difference in sharpness of the experimental curve in the stretching range (both stresses $\sigma_{x x}$ and $\sigma_{y y}$ are positive). In fact, the ratio of the experimental average plane strain tension yield stress to the uniaxial tension yield stress either in the rolling or in the transverse direction is larger for the OA material than for the UA one. However, the ratio of the balanced biaxial yield stress $\left(\sigma_{\mathrm{xx}}=\sigma_{\mathrm{yy}}\right)$ measured experimentally at the pole of the bulge specimen to the uniaxial yield stress is smaller for the 7075-OA alloy sheet. Actually, the approximation (Equ. 2) of the experimental stress-strain curves, extrapolated to larger strains, Fig. 4, gives the same trend. As a result, even though the experimental yield surface data is not very accurate, it seems that the 7075-OA and 7075-UA yield surfaces are not identical, the latter being sharper in the biaxial stretching range than the former. This difference can be directly attributed to the precipitate effects on the deformation of the UA and OA alloys since the grain morphology and the average texture are the same for the two materials.

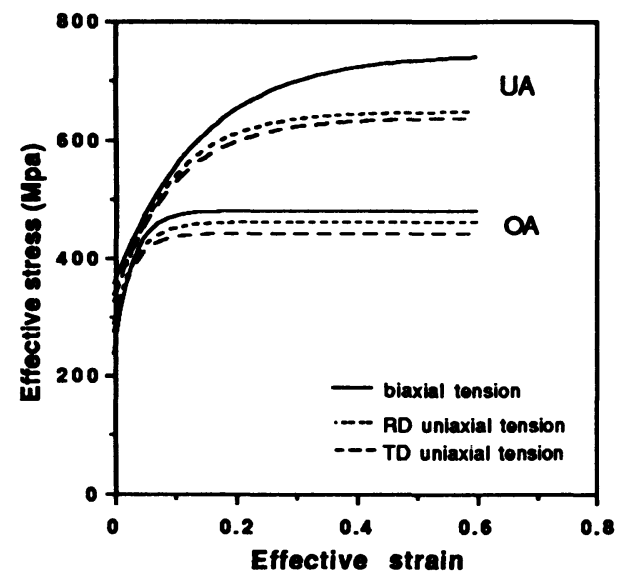

Figure 4. Effective stress vs. effective strain, Voce approximation (Equ. 2), for 7075-UA and 7075-OA in uniaxial tension in the rolling and in the transverse directions, and in balanced biaxial tension.

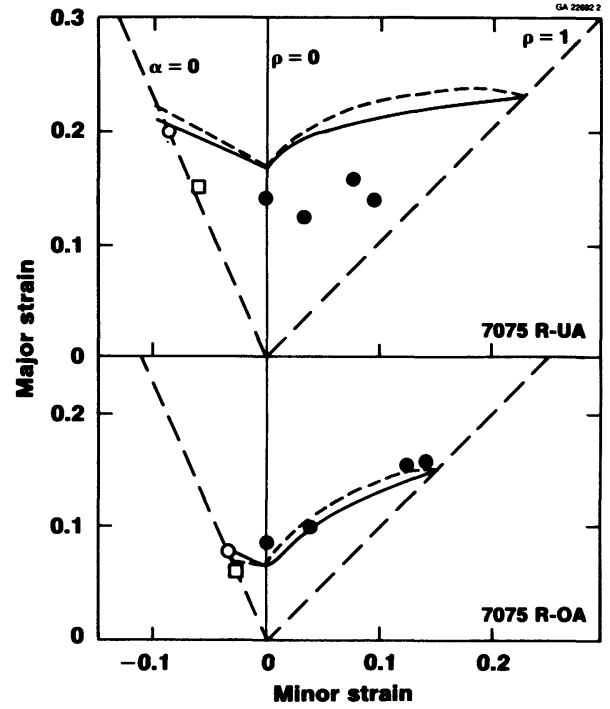

Figure 5. Experimental and theoretical forming limit diagrams (FLDs) for 7075 alloy. Major strain in the rolling direction (- - ) and in the transverse direction (-).

\section{FORMING LIMITS AND FRACTURE}

As previously mentioned, even though the yield surfaces cannot be measured directly with reasonable accuracy with the available testing machines, yield surface shape 
can be estimated from its effect on the forming limit diagram (FLD) data. It has been shown ${ }^{8,9}$ that the formability of sheet metal depends strongly on the shape of the yield surface in the biaxial stretching range where both surface strains are positive. In particular, using the Marciniak and Kuczynski (MK) analysis ${ }^{10}$ for sheet necking, it has been shown that the $R$ value is a poor parameter to evaluate stretch forming ability, whereas, the ratio $P$ of the plane strain yield stress to the equal biaxial yield stress $\mathrm{P}=\sigma_{\mathrm{p}} / \sigma_{\mathrm{b}}$ gives better formability predictions. A large $\mathrm{P}$ ratio is associated with a high balanced biaxial forming limit. This parameter $P$ is large for a Von Mises material $(\mathrm{P}=1.15)$ and small for a Tresca material $(\mathrm{P}=1)$. Consequently, the FLD for a Von-Mises material is very high for the balanced biaxial stress state whereas it is very low for a Tresca material.

Fig. 5 shows the FLD for the 7075 sheet in both UA and OA conditions. The open squares and circles represent the uniform and total elongations in uniaxial tension along the rolling direction. On the same figure, theoretical predictions computed with the MK analysis and the TBH yield surfaces are represented. The work-hardening curves measured during the bulge test have been used for expanding the yield surface isotropically during deformation. This plot clearly shows that the FLD of the OA material is very well predicted, compared to that for the UA alloy. The difference in FLD predictions cannot be entirely explained by the difference in work-hardening, since using the same work-hardening curve for both alloy conditions would lead to the same calculated forming limit. However, it is clear that the experimental FLD shapes are different. Therefore, it appears that the TBH yield surface gives a good prediction of the plastic behavior for 7075-OA sheet but not for 7075-UA, highlighting the importance of precipitate microstructure. This can be discussed in terms of the yield surface shape. The ratio of the plane strain to the biaxial yield stress seems to be larger for the OA sample. Therefore, the relative stress difference between the equal biaxial state and the plane strain state (the state that occurs at necking) is larger for the OA material. This acts like additional hardening and has been called "yield surface shape hardening"8. This yield surface shape effect compensates for the lack of work-hardening in the OA material. Therefore, the forming limit for this material is similar to the one for the UA material near the balanced biaxial stretching range.

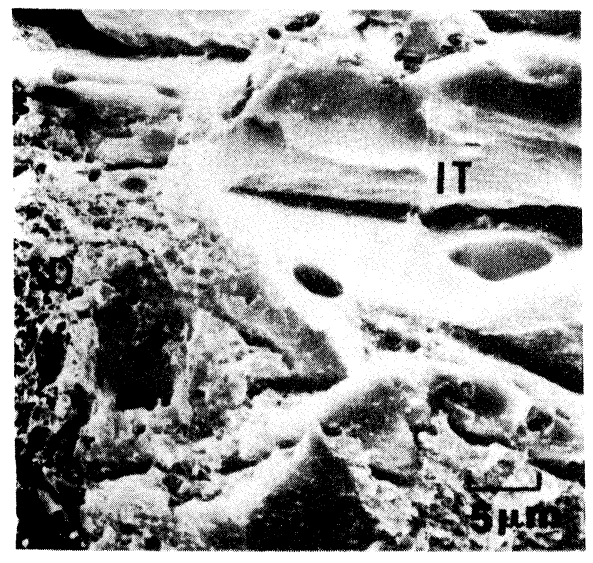

a

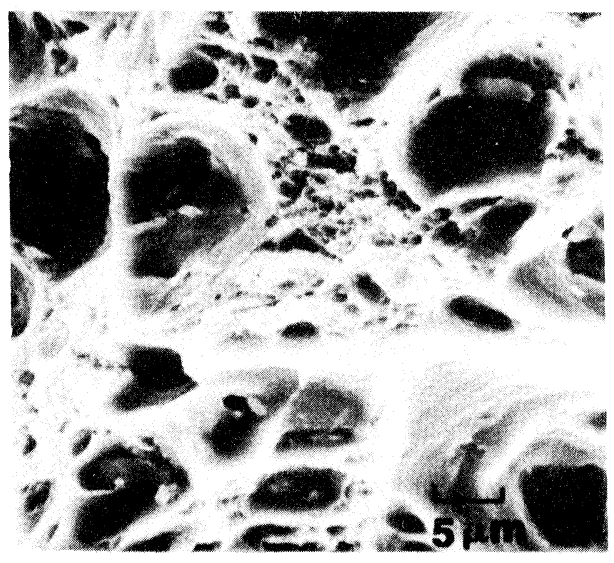

b

Figure 6. SEM fractograph of 7075 aluminum alloy; a) underaged; b) overaged 
The previous analysis concerning the yield surface shape effect on the FLD assumes that the FLD shape is not affected by fracture. Actually, fracture limits, determined from strain measurements at the fracture surface, can be represented on the same diagram, and the fracture limit curve is usually higher than the necking limit curve. However, for some aluminum alloys, it has been observed that near the balanced biaxial stretching range, these two curves intersect ${ }^{11}$. In such cases, fracture is the dominating mechanism limiting the stretchability of the material. The experimental forming limit curve is then a combination of the necking and the fracture limits. The different fracture modes commonly found in aluminum alloys ${ }^{11}$ were observed in this study. The fracture surface of the UA alloy consisted of fine microvoids on the macroscopic fracture plane (Fig. 6-a) and intergranular microvoids (IT) transverse to the macroscopic fracture plane. The overall fracture of the UA alloy resulted in a shear fracture (Fig. 6-b). Fracture surface of the OA alloy exhibited a fibrous type of failure which consisted mainly of microvoid coalescence, with dimple sizes ranging from extremely coarse (due to constituent particles) to fine. However, the fact that UA and OA microstructures differ in fracture topography need not imply differences in their FLD. In fact, some authors argue that the fracture phenomenon occurs before plastic strain localization only for materials containing very large inclusions ${ }^{12}$. As a result, in addition to fracture conditions, the yield surface shape provides an alternate or extended interpretation of the failure mechanisms near balanced biaxial tension.

\section{ACKNOWLEDGEMENTS}

The authors would like to thank Mr. D. J. Lege and Mr. J. C. Brem, Alcoa Laboratories, for the help in the analysis of experimental data. One of us (AKV) would like to thank Dr. Dale Meyn, NRL for SEM work

\section{REFERENCES}

1. W. F. Hosford and R. H. Zeisloft, Metal Trans., 3, 113 (1972).

2. P. Bate, W. T. Roberts and D. V. Wilson, Acta Met., 29, 1797 (1981).

3. M. A. Przystupa, A. K.Vasudévan and W. G. Fricke, Jr., Proc. 8th Conference on Texture of Materials, (The Metallurgical Society, 1988), p. 1051.

4. G. I. Taylor, J. Institute of Metals, 62, 307 (1938).

5. J. F. W. Bishop and R. Hill, Philos. Mag., 42, 414 and 1298 (1951).

6. J. M. Howe, J. Lee and A. K. Vasudévan, Met. Trans., 19A, 2911 (1988).

7. R. H. Wagoner, Metal. Trans., 11A, 165 (1980).

8. J. Lian, F. Barlat and B. Baudelet, Int. J. Plasticity, 5, 131 (1989).

9. F. Barlat, Forming Limit Diagrams: Concepts, Methods and Applications, (The Metallurgical Society, 1989), p. 275.

10. Z. Marciniak and K. Kuczynski, Int. J. Mat. Sci., 9, 609 (1967).

11. J. D. Embury, Proc. Int. Symposium on Aluminum Transformation Technology and Applications, (ASM, 1980), p. 141.

12. B. A. Parker, Aluminum Alloys - Contemporary Research and Applications, (Academic Press, NY, 1989) p. 539. 